\title{
Interactive Television Research Opportunities
}

\author{
Alcina Prata \\ Polytechnic Institute of Setúbal (IPS), Portugal
}

\section{INTRODUCTION}

There is no doubt that interactive TV (iTV), which may be defined as a TV system that allows the viewer to interact with an application that is delivered simultaneously, via a digital network, in addition to the traditional TV signal(Perera, 2002), will replace traditional passive TV viewing habits. In fact, this technology enables a wide range of new interactive services, applications, and features that are becoming increasingly successful. In regard to interactive services, we have the traditional iTV service (which implies interacting with an application that is simultaneously broadcasted along with the TV program), the electronic program guide (EPG) which allows the management of the enormous amount of available channels/programs and the easy selection of them based on different criteria (title, author, date, time, genre, etc.), and Internet services which include e-mail, chat, WWW, shopping, banking, and so forth. As far as iTV applications are concerned, and following Livaditi, Vassilopoulou, Lougos, and Chorianopoulos (2003), it is possible to identify four basic categories of content: entertainment (content associated with films, series, and quizzes); information (content associated with news of all kind); transactions (content used to order/purchase goods), and communication (content that involve or require the exchange of messages).

The success of iTV has mostly been due to the possibility of using different kinds of services, applications, and features through a unique and trustable device such as TV. Considering that European Internet penetration rates of around $40-60 \%$ and TV penetration rates of around $95-99 \%$ (Bates, 2003), we may anticipate a bright future for this new technology. However, as happens with any recent and emergent area, in spite all the advantages, there are many difficulties to overcome and research to be carried out. The main goal of this article is to bring together in one single source the most important research opportunities associated with
iTV and, in some cases, present specific suggestions for future developments.

For the purpose of this article, it is assumed that the person who interacts with an iTV system may be considered as a viewer (when viewing a traditional TV program and from a mass communication perspective) but also a User (when using the iTV application and from a Human Computer Interface-HCI-perspective). Thus, henceforth those who interact with iTV will be designated as Viewers/Users (V/Us).

\section{ITV RESEARCH OPPORTUNITIES}

In spite of the fact that many research groups have worked on iTV development in the last 20 years, iTV is a recent phenomenon in terms of use. Many trials were launched all around the world, but the main concern was technological.Application design has been technology-driven instead of being user-centred and has been based on the desktop paradigm instead of iTV specific guidelines. In this way, the quality of the resultant applications/services was obviously compromised. Many mistakes were made, many innovations were achieved, many lessons were learned and, more importantly, many appealing research opportunities arose from all of this. Three different research opportunity categories, in terms of their importance and urgency, have been identified. The first category refers to the main research opportunities which are, essentially, a natural consequence of the difficulties associated with iTV. The second category refers to the research opportunities that arise from the need to improve certain characteristics, which, in spite not being difficulties, may be improved. Finally, the third category, which refers to research opportunities related to completely new developments, characteristics or services. Concluding, because all the identified research opportunities need to be addressed, sooner or later, and to some extent, they have been included in this work. 


\section{First Research Opportunities Category}

Due to the lack of specific and widely accepted guidelines for the development of iTV applications (Ali \& Lamont, 2000; Chorianopoulos, 2004; Daly-Jones \& Carey, 2001; Gill \& Perera, 2003; Perera, 2002; Prata, 2005; Prata, Guimaraes, Kommers, \& Chambel, 2006) it is very important and urgent to start investigating $\mathrm{V} / \mathrm{U}$ interface principles, guidelines, rules, methodologies, and conceptual models specific for the development and evaluation of iTV applications. Also needed are methodological frameworks for defining and evaluating the quality of iTV V/U interfaces (Chorianopoulos \& Spinellis, 2006). In order to be appropriate for iTV, these methodologies/frameworks will have to consider both the motivational and emotional dimensions (Chorianopoulos et al., 2004; Springett, 2005). Furthermore, it is important to mention that iTV producers have specifically asked usability professionals to concentrate their research efforts in these areas in order to help drive the design of future interactive programs (Ali \& Lamont, 2000).

It is also very important to find ways of applying universal design in order to implement applications/ services capable of accommodating the large and different range of $\mathrm{V} / \mathrm{U}$ needs and requirements. This will enable the adequate applications/services to specific $\mathrm{V} / \mathrm{Us}$ groups, such as the elderly and impaired. Also very important is to find ways of implementing what is called "smooth interactivity," that is to say, interactivity which, in spite of being an added value to the application, does not conflict with the passive and relaxing characteristics of the media and thus may become more attractive to $\mathrm{V} / \mathrm{Us}$; interactivity which, imply minimal interference and intrusiveness; and finally, interactivity which is not too disturbing. However, considering that people are attracted to a high level of functionality, it will be important to discover how to combine high levels of functionalities with "smooth interactivity" and easiness of use. Simultaneously, it will be important to define strategies in order to demystify the idea that iTV is difficult to use. It is also important to research how to employ HCI theory and methods to the iTV field, new ways of implementing user-centred design approaches to the development of iTV applications/ services, ways of designing/developing successful iTV applications and, finally, researching the technology associated with the service in order to overcome the more common technological problems/constraints (the frequent necessity to restart the system, problems with the password, slowness in accessing applications, etc.) (Counterpoint, 2001).

\section{Second Research Opportunities Category}

It is important to carry out research in order to improve some aspects. For instance, due to the number of available channels (sometimes up to 500) EPGs need to be improved in order to become clever navigation models (Marshall, 2004). Because the EPG helps V/Us build a mental map of the order of channels they should be customizable (Perera, 2002). Due to technological and social advances, e-learning, m-learning, t-learning, edutainment, cross-device systems, and ubiquitous systems, among others, are no longer part of our imagination but, instead, are part of our lives. Thus, applications/services should be designed to be viewed through multiple platforms/devices (Marshall, 2004; Prata, 2005). However, designing interfaces for crossdevice systems is a complex task and a major challenge to designers. In fact, several devices are being used, each one with its own input/output characteristics, and thus, different device combinations imply new input/output characteristics to study. It is also important to understand how each device's characteristics (individually and when combined) influence V/Us tasks, interactivity, states of mind, and so forth (Robertson, Wharton, Ashworth, \& Franzke, 1996). Another important aspect is that different $\mathrm{V} / \mathrm{Us}$ have different characteristics in terms of skills, goals, attitudes, and so forth. Thus, personalized applications/services should be developed in order to accommodate a broad range of $\mathrm{V} / \mathrm{U}$ profiles (Marshall, 2004). Special and further research needs to be carried out regarding particular categories of V/Us, namely the elderly and those with some sort of disability (Gill \& Perera, 2003). For such groups, the online shopping service may be the only way of shopping "outside home" (Gill \& Perera, 2003; Kaye, 2000). Thus, the service should be prepared in order to accommodate these groups of special $\mathrm{V} / \mathrm{Us}$ and in this way contribute to improving their quality of life (Perera, 2002). As another example, the EPG is clearly a very useful feature that obviously is inaccessible to blind people. Some experiments were carried out, such as a virtual interface for a set-top agent (VISTA) at the University of London and Victoria University, Manchester. The idea was to make a platform indepen- 
4 more pages are available in the full version of this document, which may be purchased using the "Add to Cart" button on the product's webpage:

www.igi-global.com/chapter/interactive-television-researchopportunities $/ 17477$ ?camid $=4 \mathrm{v} 1$

This title is available in InfoSci-Books, InfoSci-Multimedia Technologies, Business-Technology-Solution, Communications, Social Science, and Healthcare, InfoSci-Media and Communications. Recommend this product to your librarian: www.igi-global.com/e-resources/library-recommendation/?id=1

\section{Related Content}

Architectural Support for Mobile Context-Aware Applications

Patrícia Dockhorn Costa, Luís Ferreira Pires and Marten van Sinderen (2006). Handbook of Research on Mobile Multimedia (pp. 456-475).

www.igi-global.com/chapter/architectural-support-mobile-context-aware/20983?camid=4v1a

A Texture Preserving Image Interpolation Algorithm Based on Rational Function Hongwei Du, Yunfeng Zhang, Fangxun Bao, Ping Wang and Caiming Zhang (2018). International Journal of Multimedia Data Engineering and Management (pp. 36-56).

www.igi-global.com/article/a-texture-preserving-image-interpolation-algorithm-based-on-rationalfunction/201915?camid=4v1a

A Generic Adaptation Framework for Web-Based Hypermedia Systems Alexandros Paramythis and Constantine Stephanidis (2005). Adaptable and Adaptive Hypermedia Systems (pp. 80-103). www.igi-global.com/chapter/generic-adaptation-framework-web-based/4180?camid=4v1a

Performance Evaluation of Relevance Feedback for Image Retrieval by "Real-World" Multi-Tagged Image Datasets

Roberto Tronci, Luca Piras and Giorgio Giacinto (2012). International Journal of Multimedia Data Engineering and Management (pp. 1-16).

www.igi-global.com/article/performance-evaluation-relevance-feedback-image/64628?camid=4v1a 